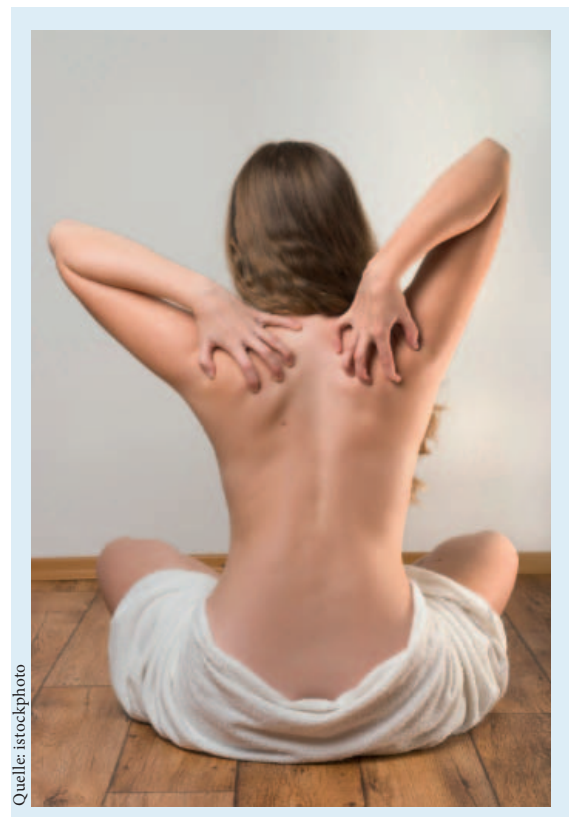

\section{Dermatologie}

Die Ganzheitsmedizin bietet eine ganze Reihe von Möglichkeiten, Hauterkrankungen zu behandeln. Im Expertengespräch auf Seite 138 zeigen Vertreter der Homöopathie und der Chinesischen Medizin die Grundzüge ihrer jeweiligen Behandlungsstrategien auf. Welchen positiven Einfluss eine Therapie im Hochgebirgsklima haben kann, erfahren Sie auf den Seiten 142 und 143. Und welche Chancen die Phytotherapie bei der Wundheilung eröffnet, haben wir auf den Seiten 134-136 für Sie zusammengefasst.

\title{
Mit Pflanzen Wunden versorgen
}

Kamille (Matricaria chamomilla) (Abb. 1), Ringelblume (Calendula officinalis) (Abb. 2) und Virginische Zaubernuss (Hamamelis virginiana) (Abb. 3) sind die wichtigsten Heilpflanzen für die Behandlung von Verletzungen der Haut. Daneben hält die Phytotherapie noch eine Reihe weiterer pflanzlicher Wirkstoffe bereit. Sie eignen sich auch für den Einsatz bei chronisch offenen Wunden.

In der Schweiz leiden etwa eine halbe Million Menschen an chronischen Wunden. Für die Behandlung bieten sich verschiedene Methoden der Komplementärmedizin an. Vor allem die Phytotherapie ist geeignet, um bei der Wundheilung eingesetzt zu werden. Schliesslich gibt es schon in der Volksmedizin eine ganze Reihe von Pflanzen, die seit langem verwendet werden, um offene Wunden besser heilen zu lassen. Sie bieten durch ihre Wirkstoffe viele Möglichkeiten, um in die biologischen Prozesse der Wundheilung einzugreifen und sie positiv beeinflussen zu können.

Die Wundheilung ist ein komplizierter physiologischer Prozess. Bei ihm kann es durch Umweltbedingungen, Infektionen mit Bakterien und Pilzen, chronische Erkrankungen des Patienten oder auch dessen fortgeschrittenes Alter zu Störungen bei der Heilung einer Verletzung kommen. Statt schnell abzuheilen, bleiben die Wunden offen. Vor allem bei älteren und bettlägerigen Patienten entwickeln sie sich zu einem gravierenden Problem. Aber auch zu Beginn des Lebens können Menschen unter Hautproblemen leiden: Bei Säuglingen kommt es durch das feuchtwarme Klima unter den Windeln sehr oft zu Hautreizungen. Auch sie lassen sich mit verschiedenen Pflanzenpräparaten gut behandeln.

\section{Pflanzen helfen bei der Wundheilung}

Es überrascht nicht, dass die Phytotherapie bei der Behandlung von Wunden eine grosse Rolle spielen kann. Die Heilung einer Verletzung der Haut ist ein sehr komplexer Prozess, bei dem eine ganze Reihe von Faktoren, die bereits als "Angriffspunkte» für phytotherapeutische Wirkstoffe bekannt sind, eine Rolle spielt. Es gibt inzwischen gut dokumentierte Fälle, in denen das Wundmanagement mit gutem Erfolg ausschliesslich aromatherapeutisch durchgeführt wurde [1].

Auch komplexe Erkrankungen lassen sich mit phytotherapeutischen Methoden behandeln. Beim Ulcus cruris, dem eine chronisch venöse Insuffizienz zugrunde liegt, sind phytotherapeutische Verfahren auch bewährt. Neben der richtigen Versorgung des Patienten durch das Tragen von Kompressionsstrümpfen bietet sich eine naturheilkundliche Lokaltherapie an: Der Ulkusgrund muss gereinigt werden. Hierfür bietet sich Kamille an. Weitere Infektionen müssen durch das Auflegen und Teilbäder mit Kamillenblüten oder Eichenrinde verhindert werden. Wenn die Infektionen sehr stark sind, haben sich auch Anwendungen mit Ringelblume oder Johanniskraut bewährt. Honig scheint ebenfalls eine gute Wirkung zu haben, da er den Bakterien durch die Osmose Wasser entzieht und so ihre Vermeh-

\section{KARGER}


rung hemmt. Um die Granulation und die Bildung von Epithelzellen zu fördern, sind Umschläge aus Kamillenblüten, Ringelblume oder Johanniskrautöl förderlich. Für kurze Zeit können auch Auflagen aus Beinwellwurzelextrakt an den Wundrand gelegt werden [2].

Die antioxidative Wirkung des Aloe-vera-Gels trägt zum Schutz der Haut bei. Ihm wird auch eine positive Wirkung bei der Wundheilung zugeschrieben. Studien zeigen, dass Aloe-vera-Gel direkt die Wirkung von Makrophagen und Fibroblasten stimuliert, was zu einer vermehrten Bildung von Kollagenen und Proteoglykanen führt, die vor allem in den ersten beiden Phasen der Wundheilung von Bedeutung sind [3].

Beinwell ist eine alte Heilpflanze, die vor allem bei Knochenbrüchen eingesetzt wurde. Sie sorgt im Allgemeinen für eine Linderung von Schmerzen sowie den Rückgang von Schwellungen und unterstützt so den Heilungsprozess von Wunden [4]. Welche Stoffe im Beinwell für die gute Wirkung bei der Wundheilung und Geweberegeneration verantwortlich sind, ist noch nicht vollständig geklärt. Allantonin und Rosmarinsäure spielen wahrscheinlich eine zentrale Rolle. Die Rosmarinsäure ist eine Phenolkarbonsäure, die entzündungshemmende Eigenschaften hat, die wahrscheinlich auf eine Inaktivierung von Komplementen zurückgeführt werden können [3].

Mit Johanniskraut lässt sich die Wundheilung fördern, und es trägt zur guten Narbenbildung bei [4]. Es wird auch bei der Behandlung von Gürtelrose verwendet. Zur Pflege von spröder und trockener Haut wird es ebenfalls eingesetzt [5].

Arnika besitzt eine hervorragende entzündungshemmende Wirkung und kann bei nicht blutenden Wunden und anderen Verletzungen eingesetzt werden. Direkt auf dem Infektionsherd wirkt Arnika ebenfalls. Ihre Wirkstoffe sind schmerzstillend und fördern die Durchblutung. Ein grosser Teil der Wirkung ist auf den Einfluss von Arnika auf die Entzündungsprozesse im Körper zurückzuführen. Es gilt als «natürliches Kortison». Verletzungen der Haut, wie Abszesse, Furunkel oder Beingeschwüre, werden mit Arnikatinktur gereinigt und mit einer in Arnikatinktur getränkten Kompresse behandelt.

Der Zwiebel konnte in einer randomisierten, placebokontrollierten Studie mit 59 Patienten ein positiver Effekt auf die Narbenbildung bescheinigt werden. Die Patienten waren operiert worden, und es zeigte sich, dass nach 3 Wochen Heilungszeit die anschliessende Anwendung eines Zwiebelextrakts über 10 Wochen die Weichheit, die Textur und das allgemeine Aussehen der Narbe verbessert hat [6].

Das Kraut und die Wurzel des Roten Sonnenhuts können topisch als verdünnter Extrakt zur Förderung der Wundheilung bei oberflächlichen Hautverletzungen angewendet werden [3].

Lavendel gilt als eines der am besten wirkenden Öle bei Wunden oder Verbrennungen. Es lindert den Schmerz und begünstigt eine narbenfreie Wundheilung [4]. Es ist das einzige ätherische Öl, das pur aufgetragen wird. Ein Tropfen reicht bereits für die Behandlung der Grösse der Handfläche [6].

Die phytotherapeutische Behandlung von Wunden stützt sich auf drei Pflanzen: die Kamille, die Ringelblume und die Virginische Zaubernuss. Zu einer ganzen Anzahl weiterer Pflanzen gibt es Hinweise, dass sie ebenfalls zum Einsatz kommen können.

\section{Kamille}

Kamille kann in allen Phasen der Wundheilung eingesetzt werden. Sie kommt zur Anwendung bei infizierten Wunden, Rissen und auch bei Verbrennungen ersten Grades. Die Heilpflanze wirkt durch die Kombi-

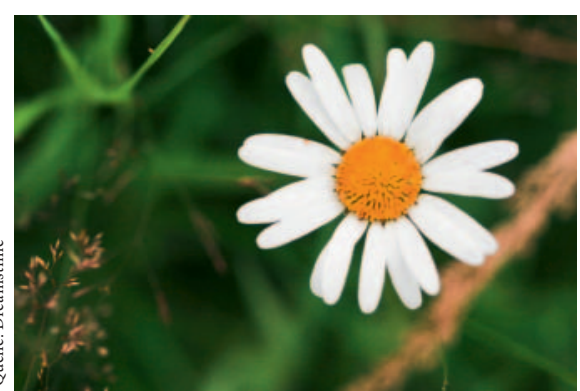

Abb. 1. Matricaria chamomilla (Kamille).

nation verschiedener Wirkstoffe, wie etwa durch Flavanoide, Schleimstoffe, also Polysaccharide, und ätherische Öle. Mit Kamillenextrakten werden juckende Ekzeme, entzündliche Hauterkrankungen und Verletzungen behandelt [7]. Die wichtigsten Inhaltsstoffe der Kamille sind Matricin und alpha-Bisabolol mit seinen Derivaten. Kamille, die in Spanien vorkommt, hat einen besonders hohen Anteil an alpha-Bisabolol [8]. Dieser Inhaltsstoff kann bei lokaler Anwendung helfen, dass andere Wirkstoffe besser durch die Haut in den Körper gelangen. Die Schleimstoffe regen die Phagozytosetätigkeit von Makrophagen und Granulozyten in den ersten beiden Phasen der Wundheilung an [3]. alpha-Bisabolol hemmt zudem das Wachstum von Bakterien und Pilzen, weshalb Kamille antibakteriell wirkt und deshalb auch bei der Therapie von schlecht heilenden, eitrigen Wunden eingesetzt wird. Kamille wirkt sich auch positiv auf den Stoffwechsel der Haut aus [4]. Sie wird ausserdem zum Auswaschen von Wundrändern, bei offenen chronischen Wunden, bei Geschwüren am Bein und bei Dekubitus eingesetzt [7]. Der Hautstoffwechsel wird angeregt und die Geweberegeneration und Entzündungshemmung werden unterstützt. Eine schnellere Wundheilung und das Abtrocknen nasser Wundflächen wurde in klinischen Studien mit standardisierten Kamillenextrakten beobachtet [3]. Einige Wirkstoffe haben eine antioxidative Wirkung [8]. 


\section{Ringelblume}

Extrakte aus Ringelblumen gehören zu den bekanntesten Mitteln der Phytotherapie, die bei der Behandlung von Wunden eingesetzt werden. Die Blüten der Ringelblume wirken entzündungshemmend und wundheilend, indem sie die Bildung von Granulationsgewebe fördern. Die Wirkstoffe, die dafür verantwortlich sind, sind wahrscheinlich Karotenoide und ihre Abbauprodukte, die chemisch dem granulationsfördernden Vitamin A nahestehen [3]. Ihre Flavonoide und ätherischen Öle hemmen das Wachstum verschiedener Bakterien [4,5]. Sie werden bei schlecht heilenden, eiternden Wunden und Geschwüren eingesetzt. In der Dermatologie sind Einsatzgebiete zum Beispiel Windeldermatitis und Ekzeme. Offene Wunden lassen sich ebenfalls mit Präparaten aus Ringelblumen behandeln [7]. Zur Hautpflege eignen sich Ringelblumenpräparate. Wirkstoffe der Pflanze werden bei empfindlicher, trockener und zu Allergien neigender Haut eingesetzt. Besonders scheint sie sich bei der Pflege von Baby- oder Altershaut zu eignen [5].

Es gibt Hinweise, dass ein Gemisch aus Ringelblumen und Johanniskraut nach einer Kaiserschnittoperation $\mathrm{zu}$ einer schnelleren Wundheilung führt. Auch nach einer Strahlentherapie geschädigte Haut verheilte mit einer Ringelblumensalbe besser als mit einem herkömmlichen Präparat [4].

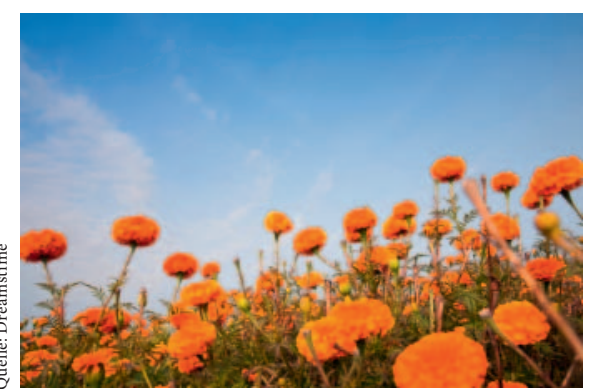

Abb. 2. Calendula officinalis (Ringelblume).

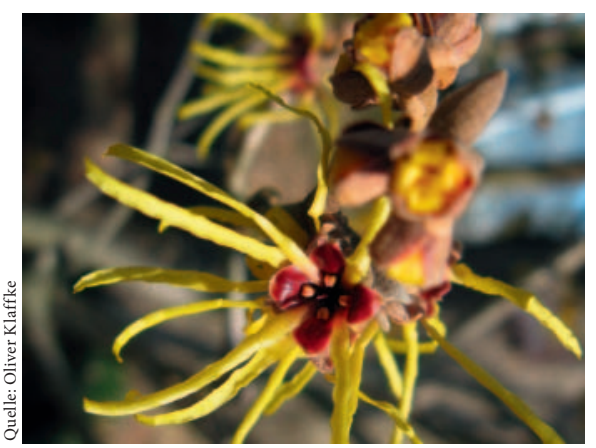

Abb. 3. Hamamelis virginiana (Virginische Zaubernuss).

\section{Virginische Zaubernuss}

$\mathrm{Zu}$ den wichtigen Heilpflanzen, die bei der Behandlung von Wunden und in der Dermatologie eingesetzt werden, gehört die Virginische Zaubernuss. Ihre Rinde enthält bis $\mathrm{zu} 12 \%$ Gerbstoffe, die Blätter bis zu etwa $10 \%$, und zusätzlich auch kleine Mengen an ätherischem Öl. Deshalb haben die unterschiedlichen Zubereitungsarten für Hamamelis-Präparate eine unterschiedliche Zusammensetzung an Inhaltsstoffen und damit auch un- terschiedliche Wirkungen zur Folge. Daher sind auch die Indikationen leicht unterschiedlich: Destillate kommen bei leichten Hautentzündungen zum Einsatz. Sie können auch in speziellen Darreichungsformen, etwa bei Irritationen des Auges durch Trockenheit, Wind oder Sonnenlicht, eingesetzt werden. Auszüge aus den Blättern und der Rinde der Zaubernuss eignen sich für die Behandlung von leichten Entzündungen der Haut oder der Mundschleimhaut, aber auch für die symptomatische Behandlung von Hämorrhoiden [9]. (rfi)

\section{Literatur}

1 Steflitsch W: Wundbehandlung mit ätherischen Ölen nach operativer Sanierung eines chronischen Abszesses. Forsch Komplementmed 2009;16:400-403.

2 Bühring U: Heilpflanzen bei Ulcus cruris. Z Phytother 2005;6:185-187.

3 Augustin M, Hoch Y: Phytotherapie bei Hauterkrankungen: Grundlagen, Praxis, Studien. München, Urban und Fischer, 2004.

4 Wösch A: Die Rolle der Natur in der Wundbehandlung. Projektarbeit. 2010. www.wundmanagement-tirol.at/files/die_rolle der_naturanja_woesch_pdf.pdf.

5 Bühring U: Alles über Heilpflanzen - erkennen, anwenden, gesund bleiben. Stuttgart, Ulmer, 2007.

6 Draelos ZD: The ability of onion extracts gel to improve the cosmetic appearance of postsurgical scars. J Cosmet Dermatol 2008;7:101-104.

7 Fischer-Rizzi S: Medizin der Erde. Baden, AT, 2010.

8 Boltshauer V: Wundheilung mit Kamille. Phytotherapie 2005;2:12-14.

9 Länger R: Zauberstrauch (Hamamelis virginiana). PHYTO Therapie Austria 2010;4:8. 